\title{
A Radar Signal Sorting Algorithm Based On Entropy Features Set Pair Analysis
}

\author{
JianFeng $\mathrm{PU}^{1, \mathrm{a}}$, Hui $\mathrm{CHEN}^{1}$,XuZhou ZHANG ${ }^{1}$, Qian $\mathrm{MA}^{1}$, XingWen DONG ${ }^{1}$ \\ ${ }^{1}$ Aviation University of Air Force, Changchun 130022, China \\ aemail: pujianfeng2002@163.com
}

Keywords: Signal Sorting;Set pair analysis;entropy features;Sorting index

\begin{abstract}
Facing the problem of the difficulty to sort the radar signal under the complex battlefield environment, this algorithm can sort the unconbentionality radar signal of jittered PRI or staggered PRI in real-time effectively with high-accuracy, using the principle of Set Pair Analysis and taking the entropy feature as its index weight in real time efficiently. The algorithm can solve the problem of confirming the value of the allowance in traditional algorithm. The result shows that: compared to the traditional algorithm, the algorithm can improve the efficiency of $5.7 \%$ clustering accuracy enhance and $16 \%$ time saving for 10 kinds of the typical radar signal.
\end{abstract}

\section{Introduction}

The radar signal sorting restricted by the following three factors: (1) a sharp increase in the number of radar, making Battlefield Environment radar signal intensity abnormalities, increasing unusually intense, increasing the difficulty of signal separation, and the serious influence the real-time performance of signal separation; (2) domestic and foreign military radar signal form has become increasingly complex, a new system radar constantly to replace the traditional regular pulse radar, further increasing the difficulty of signal separation and also restricts the signal sorting real-time [1-2], (3) the existence of various interference sources makes inevitable in reconnaissance signal mixed with noise pulse,, serious damage to the signal sorting utilized regularity, whitch proposes new challenges to modern radar signal sorting .

For example, surveillance alarm requires real-time alarm within a few hundred milliseconds. So it puts forward higher requirements for real-time sorting algorithm. Set pair analysis is a uncertainty analysis method of quantitative analyzing an uncertain new coupled system [9], this paper tries to treat radar signal sorting system under complex electromagnetic environment as a uncertainty system, sets pair of radar reconnaissance receiver machine received radar pulse signal and pre-existing radar pulse in Knowledge base, then classifies by calculation of department of different reverse connection degree, in order to achieve separation of radar signals.

\section{Set Pair Analysis Theory}

In the system certainty is divided into the "same" and "opposition" aspects. Uncertainty is called "difference". He analyzes things from three aspects as same, different, and opposition. The Same, different and opposition interrelate, influence, restrict each other , and mutual transform under certain conditions, [10]. By introducing connection degree and its mathematical expression to describe various uncertainties, then uncertainty of dialectical logic can be converted to mathematical operations.

Set pair is made of the connection of two collections in pairs. The basic idea is: in the context of specific question, The characteristics of set pair consisting of A and B are analyzed to give a total of $\mathrm{N}$ features,.there are $\mathrm{S}$ characteristics have in common between the two collections, the other $\mathrm{P}$ characteristics are opposite. the rest of $\mathrm{F}$ characteristics are not sure, the two collection of 
connection degree is:

$$
\mu=\frac{S}{N}+\frac{F}{N} i+\frac{P}{N} j=a+b i+c j, a+b+c=1
$$

Type: ${ }^{\mu}$ is link degree; $\frac{S}{N}$ is the same degree of set A and set B, shorthand for ${ }^{a}$;

$\frac{F}{N}$ is the different degree, shorthand for $b ; \frac{P}{N}$ is the opposite degree; shorthand for ${ }^{c} ; i$ is the different tag, Its value is in the interval[$[-1,1]$ according to the different conditions. It can also plays a role of tag only; $j$ is the coefficients of opposites. Its value is -1.It can also only tag role.

It can be seen, the expression of link degree reflects the same, differences, opposition contacts, influence and transformation at the same time, When $i=1$, the different degree is converted into the same degree; when ${ }^{i=-1}$ it is transformed into opposite degree; when ${ }^{(-1,1)}$ the value in the interval $^{(-1,1)}$, the same degree and opposite degree each account for a certain proportion. Contact degree ${ }^{\mu}$ and uncertainty factor $i$ is the cornerstone of the theory. The theory can describe the random, fuzzy, gray and other common uncertainty phenomenon.

\section{The entropy feature extraction}

Entropy is the characteristic parameter of a disordered state, often used to measure disorganized, unbalanced, uncertainties and other issues. Entropy features include approximate entropy ( ApEn) feature and norm entropy (NoEn) feature. Approximate entropy is a quantitative description of signal complexity and irregularity .Norm entropy is a valid quantitative characterization of signal energy distribution.

\section{(1) approximate entropy (ApEn) extraction and calculation}

ApEn is a statistical parameters ,which is a quantitative description of the time series complexity and irregularity .It does not have to reconstruct all the strange attractors, just use less data statistical probability of having a different size complexity of the signal sequence to produce a new model , so it has better noise suppression.

ApEn characteristic values of radar signal can be calculated using the following steps to extract :

Step 1: signal preprocessing

The receiver intercepts a signal of a radiation source $\left\{f(i), i=1,2, \cdots, M_{1}\right\}, M_{1}$ is the length of the signal sequence) can be transformed from the time domain to the frequency domain by Fourier transform, so that the extracted features are not affected by changes in carrier frequency signal. Because the signal amplitude spectrum is symmetrical, so only consider the magnitude of the right of the spectrum $\left\{f(i), i=1,2, \cdots, M_{2}\right\},\left(M_{2}=M_{1} / 2\right)$ 。

Step 2: Normalized

Normalizing the power of the signal $\{f(i)\}$, then obtain the signal $\left\{R(i), i=1,2, \cdots, M_{2}\right\}$ 。

Step 3: Approximate entropy computing

Let the vector of dimension is $\mathrm{m}$ ， the noise tolerance is $\mathrm{r}$, calculate ApEn value:

(1) Form the signal sequence $\{R(i)\}$ to a group m-dimensional vector, that is,

$X(i)=(R(i), R(i+1), \cdots, r(i+m+1))$,

$j=1,2, \cdots, N-m+1$. 
(2)Calculate the distance between each value $i$ vector $X(i)$ and the rest of the vector $X(j)(j=1,2, \cdots, N-m+1$, 且 $j \neq i)$

$d[X(i), X(j)]=\max \{|R(i+k)-R(j+k)|\}, \quad k=1,2, \cdots, m-1$.

(3) Calculate the number of each value $j \quad d[X(i), X(j)]<r \quad(\mathrm{r}(\mathrm{r}>0)$ is noise tolerance, the values $i$ is the same to step 2), notes for $C_{n}^{m}(j)$, calculate the ratio of it in to the total number of vector $N-m+1$ :

$$
C_{j}^{m}(r)=\frac{1}{N-m+1} C_{n}^{m}(j)
$$

(4)Taking the natural logarithm of $C_{j}^{m}(r)$, and then average for all $\mathrm{j}(\mathrm{j}=1,2, \ldots, \mathrm{N}-\mathrm{m}+1)$, as $\Phi^{m}(r)$, namely:

$\Phi^{m}(r)=\frac{1}{N-m+1} \sum_{j=1}^{N-m+1} \ln \left[C_{j}^{m}(r)\right]$

(5) the vector dimension m increase 1 , into $\mathrm{m}+1$, calculate $C_{j}^{m}(r)$ and $\Phi^{m}(r)$ through the steps $(1) \sim(4)$.

(6) Calculate signal ApEn value:

$$
\operatorname{ApEn}=\Phi^{m}(r)-\Phi^{m+1}(r)
$$

According to the literature, take $m=2, r=0.15 S T D$, STD is the standard difference of the signal sequence $\{R(i)\}$, this time approximate entropy has a more reasonable statistical properties.

\subsection{Norm entropy characteristics (NoEn) extraction and calculation}

The spectral shape, energy distribution and concentration of different systems radar signals are different . Norm entropy NoEn just can quantitatively describe the energy distribution of the radar signal.

Definition 1: Let signals,

$$
\begin{aligned}
S= & \{s(i), i=1,2, \cdots, N\}, E_{i}=|s(i)|^{p},(1<p<2) \\
& N o E n=\sum_{i=1}^{N} E_{i}
\end{aligned}
$$

the norm entropy of signal S.

NoEn feature value of the radar emitter signal can be extracted and calculated using the following steps ::

Step 1: signal preprocessing

The receiver intercepts a signal of a radiation source $\left\{f(i), i=1,2, \cdots, M_{1}\right\} \quad{ }^{M_{1}}$ is the signal sequence length) can be transformed this.

$\left\{f(i), i=1,2, \cdots, M_{2}\right\}, \quad\left(M_{2}=M_{1} / 2\right)$

Step 2:denoising 
The de-noising process can be described as

$$
f_{d}(i)= \begin{cases}f_{t}(i)-M_{f}, & f_{t}(i)>M_{f} \\ 0, & f_{t}(i) \leq M_{f}\end{cases}
$$

Where: $M_{f}$ is the Mean of signal $f_{t}(i)$.

Step 3: Normalized

The signal $f_{d}(i)$ is normalized to obtain a processed signal $\left\{f_{p}(i), i=1,2, \cdots, N\right\}$.

Step 4: Norm entropy computing

NoEn value can be calculated based on a formula that

$$
\operatorname{NoEn}\left(f_{p}(i)\right)=\sum_{i=1}^{N} E_{i}=\sum_{i=1}^{N}\left|f_{p}(i)\right|^{p}
$$

\section{Signal sorting analysis based on entropy feature set}

In this paper, the concept of characteristics entropy is introduced, approximate entropy (ApEn), Norm entropy (NoEn), DOA parameters are used to clustering sorting, that can achieve good results in the initial sorting.

The step of cluster analysis using Set pair analysis is as follows:

(1) when the radar warning received space radar pulse data, at first ,taking a small string of pulses (for the experiment, it generates a total of $100 \times 10$ pulses, taking the first 1000 pulses) to sort using grid clustering algorithm . the detail of the algorithm can be found in [7]. The sorted radar was seen as known radar Knowledge.

$R=\left\{R_{1}, R_{2}, R_{3}, \cdots R_{N}\right\}$

$\mathrm{N}$ radars are seen as a possible classification set, each radar corresponds a feature vector $R_{i}$.

$R_{i}=\left\{\mathrm{ApEn}_{\mathrm{i}}, \mathrm{NoEn}_{\mathrm{i}}, D O A_{i}\right\}^{T}, \quad R_{i} \subset R$

(2) the subsequent arrival of the first pulse $\mathrm{P}$, calculate the feature vector

$P=\left\{\mathrm{ApEn}_{\mathrm{P}}, \mathrm{NoEn}_{\mathrm{p}}, D O A_{\mathrm{P}}\right\}^{T}$

set pair of the p and known radars , their characteristic parameters pair is as follows:

$\left(A p E n_{p}, \mathrm{ApEn}_{\mathrm{i}}\right),\left(\mathrm{NoEn}_{\mathrm{p}}, \mathrm{NoEn}_{\mathrm{i}}\right),\left(D O A_{p}, D O A_{\mathrm{i}}\right)$

(3) The system based on classification model is concerned with only three factors, the same-different Anti connection degree of the factor $t(t=1,2,3)$ is ${ }^{{ }^{\prime}}{ }^{\prime}$, you can get three connection degrees : $u_{k}^{1}, u_{k}^{2}, u_{k}^{3}$,respectively correspond ApEn, NoEn, DOA.

(4) Determine the weights of all the factors $\alpha=\left(\alpha_{1}, \alpha_{2}, \alpha_{3}\right)$, weights represent the importance of each factor, the degree of contact plays an important role. We should be reasonably determine weights.

(5) based on the $m$ connection degrees and weighting coefficient of contact factors, the connection degree can be obtained

$$
u_{k}=\alpha_{1} u_{k}^{1}+\alpha_{2} u_{k}^{2}+\alpha_{3} u_{k}^{3}=\sum_{i=1}^{3} \alpha_{i} u_{k}^{i}
$$

(6) Compute set pair potential (SHI) of the connection degree to determine the clustering results. Set pair potential is defined as

$$
\operatorname{SHI}\left(u_{k}\right)=a_{k} / C_{k}
$$

If $\operatorname{SHI}\left(u_{i}\right)=\max \left\{\operatorname{SHI}\left(u_{k}\right)\right\}$, the radar pulse is considered to be classified $\mathrm{P}$ is closest with the sort mode signal ${ }^{R_{i}}$, so $\mathrm{P}$ is included in the mode ${ }^{R_{i}}$, namely that the radiation of the pulse signal is $R_{i}$.

(7) Take the next pulse, repeat step 2. 


\section{Experimental results and Conclusion}

This effective of the algorithm on the radar signal sorting can be illustrated by experiment of five kinds of common radar signal. Extract five common types of radar signals in real electromagnetic environment, they are: 1. continuous wave signal (CW), 2 linear frequency modulation signal (LFM), 3 nonlinear frequency modulation signal (NLFM), 4 two-phase encoding. signal (BPSK) and 5. four-phase encoding signal (QPSK). Sampling parameters: carrier frequency is $50 \mathrm{MHz}$, the sampling rate is $200 \mathrm{MHz}$, the frequency offset is $5 \mathrm{MHz}$, pulse width is $0.75 \mu \mathrm{m}$, BPSK is 7 Barker code, QPSK is Taylor four-phase code. Each of the 5 radars provide 150 full radar pulse. They alias together according to the order of arrival time to form the original source signal sorting. Their traditional parameters(DOA, RF, PW, PRI) are shown in Table 1.

Table.1 Radar parameter settings

\begin{tabular}{c|cccc}
\hline & $D O A\left({ }^{\circ}\right)$ & $R F(G H z)$ & $P W(u s)$ & PRI(us) \\
\hline CW & $54 \sim 56$ & $9.7 \sim 9.9$ & $1.5 \sim 3.5$ & 80 (fixation) \\
LFM & $58 \sim 60$ & $9.6 \sim 9.8$ & $3.5 \sim 5.5$ & 70 (twitter) \\
NLFM & $55 \sim 57$ & $9.9 \sim 10.1$ & $5.5 \sim 7.5$ & {$[23,47,73]$ (irregular) } \\
BPSK & $59 \sim 61$ & $9.5 \sim 9.7$ & $7.5 \sim 9.5$ & {$[53,29,43]$ (grade) } \\
QPSK & $63-62$ & $9.6 \sim 9.7$ & $7.8 \sim 9.5$ & {$[13,23,13]$ (grade) } \\
\hline
\end{tabular}

First, Replace the traditional parameters, Extracted entropy parameters are shown in Table 2 Table 2 The new feature parameter of radar signal

\begin{tabular}{c|ccccc}
\hline \multirow{2}{*}{ Signal types } & DOA $\left(^{\circ}\right)$ & \multicolumn{2}{|c}{ ApEn } & \multicolumn{2}{c}{ NoEn } \\
\cline { 3 - 6 } & & expectation & variance & expectation & variance \\
CW & $54 \sim 56$ & 2.79261 & 3.0360 8e-004 & 0.77945 & $5.21368 \mathrm{e}-004$ \\
LFM & $58 \sim 60$ & 0.01911 & $3.25517 \mathrm{e}-004$ & 3.08212 & $5.26526 \mathrm{e}-005$ \\
NLFM & $55 \sim 57$ & 15.9793 & $1.79951 \mathrm{e}-008$ & 0.20017 & $2.06356 \mathrm{e}-002$ \\
BPSK & $59 \sim 61$ & 0.02178 & 4.0836 2e-004 & 6.25337 & $2.40234 \mathrm{e}-004$ \\
QPSK & $63-62$ & 0.32265 & $6.63148 \mathrm{e}-004$ & 7.31788 & $3.5526 \mathrm{e}-004$ \\
\hline
\end{tabular}

The effection of radar signal sorting based the traditional set pair analysis algorithm is shown in Figure 1.Use the set pair analysis algorithms of feature entropy presented in this paper to sort the same signals. The result is shown in Figure 2.

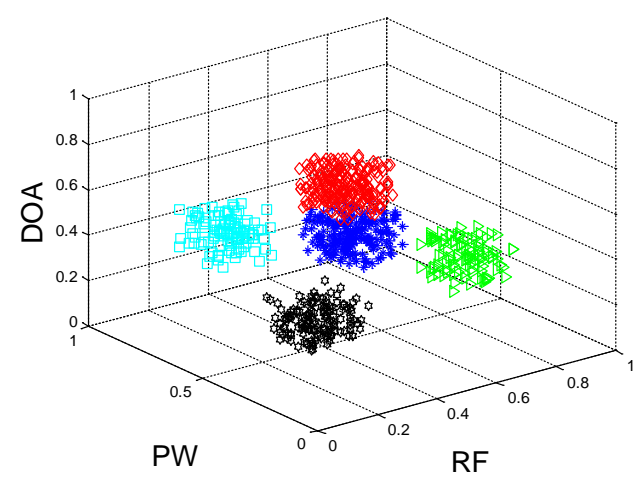

Fig.1 The effection of radar signal sorting the traditional set pair analysis algorithm

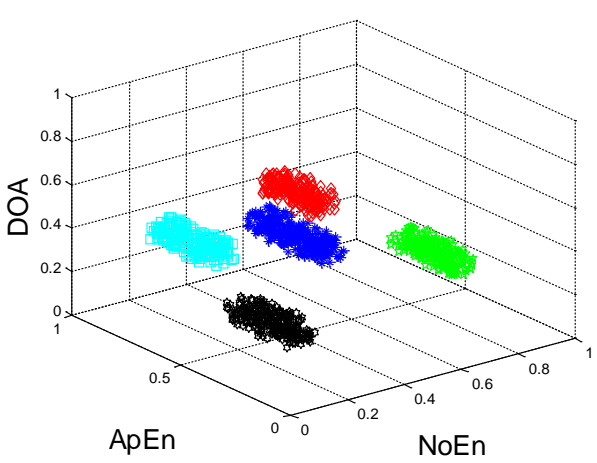

Fig.2 The effection of radar signal sorting based the improved set pair analysis algorithm

The new algorithm costs 4 943ms.Because add the characteristic entropy model, the calculation time extends more $21 \mathrm{~ms}$ than the traditional method , $0.42 \%$ of the total algorithm time. The time can be ignored.by contrasting with the technical and tactical indicators of warning device. But the 
sorting effect of the new algorithm has significantly improved .Its radar signal sorting effect is shown in Figure 3.

Defined radar pulse signal sorting correct rate: $\mathrm{CR}=$ (number of correct sorting pulses / total number of pulses) $\mathrm{x} 100 \%$. Table3 is the statistics result of the two pulse radar signal sorting algorithm .Seen from the results in Table 3, the sorting correct rate based on set pair analysis (97.83\%) is higher than the sorting correct rate of nuclear fuzzy clustering (95.65\%), and in the case where the carrier frequency is very close, there is no misclassification election situation .It describes the radar signal sorting algorithm based on set pair analysis clustering has good sorting performance.

\begin{tabular}{c|cccc} 
Table.3 & The new feature parameter of radar signal \\
\hline & $\begin{array}{c}\text { pulse } \\
\text { counts }\end{array}$ & $\begin{array}{c}\text { correct } \\
\text { counts }\end{array}$ & $\begin{array}{c}\text { accuracy } \\
\text { rate }\end{array}$ & $\begin{array}{c}\text { Paring } \\
\text { time }\end{array}$ \\
\hline $\begin{array}{c}\text { The Algorithm } \\
\text { of tradition } \\
\text { The arithmetic } \\
\text { of haracter entropy }\end{array}$ & $100 \times 10^{4}$ & 925753 & $92.5 \%$ & 143 \\
\hline
\end{tabular}

\section{References}

[1] LI He-sheng, HAN Yu, CAI Ying-WU, etc. Overview of the crucial technology research for radar signal sorting[J]. Systems Engineenng and Electronics,2005, Vo1. 27, No. 12: 2035-2040.

[2] LIU Xu-bo , SI Xi-cai . Sorting of radar-signals based on modified fuzzy clustering[J]. Journal of Projectiles,Rockets,MissilesandGuidance,2009, Vo1. 29, No. 5 : 278-282.

[3] Willson, Gregory B. Radar classification using a neural network[J]. Public by Int Soc for Optical Engineering, 1990, 1294: 200-210.

[4] Granger Eric, Savaria Yvon, Lavoie Pierre, Cantin Marc-Andre. Comparison of self-organizing neural networks for fast clustering of radar pulses[J]. Elsevier Sci BV, 1998, 64(3): 249-269.

[5] Mardia H K. Adaptive Clustering for ESM[J]. IEE Colloquium on Signal Processing for ESM systems, 1988, 62(5): 149-154.

[6] Milojevic D J, Popovic B M. Improved Algorithm for the Deinterleaving Radar Pulses[J]. IEE Proceedings, Part F:Radar and Signal Processing, 1992, 139(1): 98-104.

[7] Nelson D J. Special purpose correlation functions for improved signal detection and Parameter estimation[C]. Seattle: Proceedings of International Conference on Acoustics, Speech, and Signal Processing, 1993(4): 73-75.

[8] Nishinuchi K, Kobayashi M. Improved algorithm for estimating pulse repetition intervals[J]. IEEE Transactions on Aerospace and Electronic Systems, 2000, 36(2): 407-419.

[9] ZHAO Ke-qin. Set pair analysis and application- [M]. Hangzhou: Zhej iang Science and Technology Press, 2000.

[10] Fang Kaitai, Pan Enpei.Cluster Analysis[M]. Bei- jing: Geological Publishing House, 1982. 